\title{
Spectrum Sensing Using Energy Detector in Rayleigh Fading Channel
}

\author{
Hemlata Patil ${ }^{1 *}$, Sai Vennela Nekkanti ${ }^{1}$, Disha Negi ${ }^{1}$, Nikita Masanagi ${ }^{1}$, and Sakshi More ${ }^{1}$ \\ ${ }^{1}$ EXTC Department, RAIT, Nerul, India.
}

\begin{abstract}
Cognitive Radio, which is an adaptive and intelligent radio has an important feature called spectrum sensing. It monitors the presence or absence of the authorized users in a fixed or licensed spectrum. There is a rise in the use of wireless communications in recent times, which has led to the underutilization of the available bandwidth. This problem can be solved to some extent by using cognitive radio technology. Cognitive radio gives an opportunity for unauthorized users to use the licensed spectrum, resourcefully, when not in use. Using spectrum sensing energy detection methods, the existence of authorized users, in various fading channels can be done. The operating characteristics of the receiver can be analyzed using parameters of performance like signal to noise ratio, probability of false-alarm, probability of detection. In this paper we assess Rayleigh fading channel and compare with AWGN (non- fading) channel. Theoretical and simulated results are compared, and observations are being made.
\end{abstract}

\section{Introduction}

The conventional wireless networks use constant policies for spectrum allocation for authorized users. Current studies show that spectrum utilization is very inefficient using conventional spectrum allocation policies. This inefficiency arises because of the fact that authorized users do not exploit the available spectrum completely. Therefore, in order to satisfy the growing demand for communication and wireless application, the requirements for a flexible spectrum management approach are emerging to reflect the efficacy of the band. Cognitive radio is a powerful tool that can allow a dynamic approach to spectrum access and address the present inadequacy issues. It can allow unauthorized users to gain access to the available spectrum left unused by the authorized user[2].

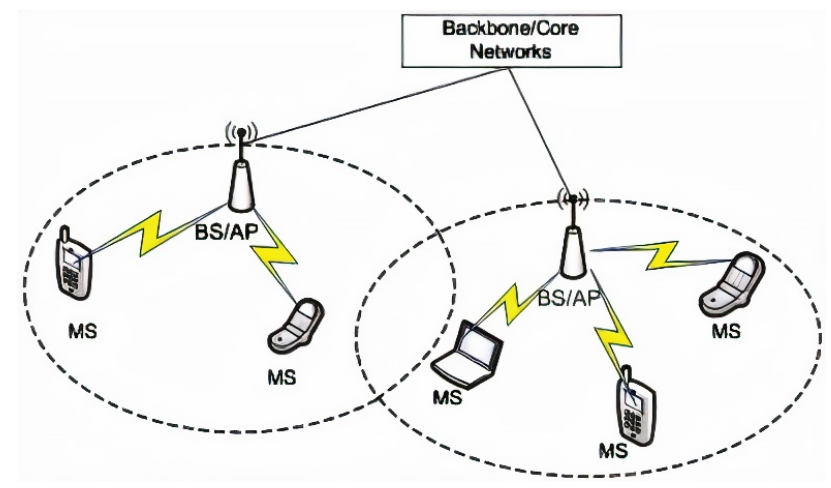

Figure 1. Cognitive Radio fundamental block diagram

Cognitive Radio technique is introduced to occupy the holes in the spectrum when it is not fully utilized and

\footnotetext{
*Corresponding author - hema.pati108@gmail.com
}

segment the spectrum avoiding damaging interference resourcefully. Hence, continued sensing of the spectrum is a significant job for sensing the existence of authorized users by CR as shown in Figure 1. Here, energy detectors are used for the measurement of received signal energy for a fixed interval of time [1].

J.Mitola. established a unique approach to the entire world about the term CR which can be used for enhancing the utilization of a valuable resource, the electromagnetic radio spectrum [1]. The idea of SDR and the abridged way to execute it was characterized by Simon Haykin. He declared that software radios are advancing stages for multimode communications [2]. Diverse spectrum sensing methods were proposed by Fabrício B. S. de Carvalho. In his theory, he explained the concept of $\mathrm{CR}$ along with the analysis of CR spectrum sensing using ED for various fading channels [3].The authors investigated the spectrum sensing method of CR rooting ED. While fulfilling dependability limitations and giving a throughput to SUs an optimization issue occurs reducing the energy utilized in sensing the spectrum. To unravel this issue an arrangement is generated to decide the ideal sensing, informing and transmission period [4]. Evaluation and simulation outcomes proclaimed the esteem value sensing, informing, and transmission period to attain the top leading trade-off amidst the consumption of energy and throughput for SUs [5]. The issue of energy detection of obscured deterministic signals of the authorized users across the Nakagami-m fading channel and presuming ECG method is examined by Herath et al [6]. The authors in analyzed the performance of ED across different fading channels like rayleigh, rician, and nakagami using diversity reception. The authors obtained theoretical results and practical results for probability detection and 
false alarm in terms of various channels [7].The authors concluded their observations that Cognitive Radio Network with low computational complications, can detect the existence of primary user signal which is the easiest job via ED based Spectrum sensing technique [8][9]. There are some limited theories on the working of ED spectrum sensing for composite multipath fading [10][11].

In this paper, we discuss spectrum-sensing techniques via energy detectors for Rayleigh fading channels and study the ability to detect signals. The mathematical expressions for Rayleigh and non-fading AWGN channels are derived including false alarm probability and the detection-probability in correlation with a signal to noise ratio.

A simulation model has been established using MATLAB aimed at spectrum sensing based on energy detection. The theoretical results are precisely synchronized with the simulated results of the Rayleigh channel and AWGN channel. Various parameters like the value of SNR, different fading parameters for different fading channels have been analyzed.

\section{System Model}

One of the significant functions for discerning wireless network communication is spectrum sensing. Continuous sensing is required for assigning cognitive radio users with the unoccupied spectrum bands. Mainly, there are 3 detection methods, to recognize the unused band which is vacant. They are namely, a) Matched-filter-detector, b) Cyclostationary-detector and c) Energy-detector [8]. Among these, the most widely used method is an energy detector, because it does not need previous information of the main signal and is less complex than other methods.

In $\mathrm{ED}$, the received signal is observed and the existence or absence of primary signal is decided. This is done by assuming that the primary signal is noise. Energydetectors are robust to the variations in the main signal. Furthermore, it has low complexity because it does not require tedious signal processing. It is preferable for widerband spectrum sensing. The functional block diagram of ED is given in Figure 2 which consists of four main parts [9].

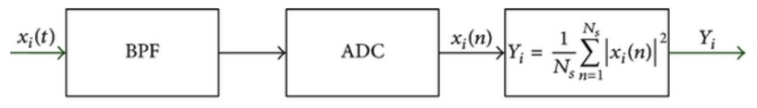

Figure 2. Functional diagram of Energy Detector

The input signal is first sent through the bandpass filter that is then sent to the squaring device and integrator.The output from the integrator is the signal which is received at the ' $\mathrm{T}$ ' time interval. This yield is then passed through the threshold device and tested for 2 hypotheses $\left(\mathrm{H}_{0} \mathrm{H}_{1}\right)$
$\mathrm{H}_{0}$ refers to the presence of only noise and absence of the signal and $\mathrm{H}_{1}$ refers to the existence of both signal and noise.

Hence the significant cases for two-state hypothesis are [9]:-

$$
\begin{aligned}
& \mathrm{Y}(\mathrm{m})=\mathrm{W}(\mathrm{m}): \mathrm{H}_{0} \\
& \mathrm{Y}(\mathrm{m})=\mathrm{X}(\mathrm{m})+\mathrm{X}(\mathrm{m}): \mathrm{H}_{1}
\end{aligned}
$$

Here, $\mathrm{X}(\mathrm{m})$ is the Input signal, $\mathrm{Y}(\mathrm{m})$ is the output, and $\mathrm{W}(\mathrm{m})$ is the noise.

a) $\mathrm{H}_{1}$ becomes true when the authorized user is not present that is nothing but false alarm probability $\left(P_{F}\right)$ $\left[\mathrm{P}\left(\mathrm{H}_{1} / \mathrm{H}_{0}\right)\right]$ b $) \mathrm{H}_{1}$ is true when the authorized user is present that is called detection-probability $\left(P_{D}\right)\left[\mathrm{P}\left(\mathrm{H}_{1} / \mathrm{H}_{1}\right.\right.$ )]. c) $\mathrm{H}_{0}$ is true when the authorized user is available is called the missed-detection probability $\left(P_{m}\right)\left[\mathrm{P}\left(\mathrm{H}_{0} / \mathrm{H}_{1}\right)\right]$ [7]

\subsection{Decision Statistic}

Decision theory or decision statistics is the part of statistics that deals with making optimal decisions, using quantitative methods in uncertain times. Let us consider the subsequent representations:

$\mathrm{x}_{i}(\mathrm{t})$ is the input signal which is the waveform which is transmitted, $y_{i}(t)$ is the output signal which is the waveform received, $\mathrm{pq}(\mathrm{t})$ is quadrature phase component, pi(t) is in phase-noise component. $\mathrm{BW}_{N}$ is noise-bandwidth, $\mathrm{N}_{0}$ is two-sided power spectral density, $\mathrm{N}$ is one-sided power spectral-density, $\mathrm{E}_{s}$ is the signal energy is the sampling interval, $\Lambda$ is decision threshold. The received-signal is filtered by a band-pass filter which is the pre-filter. The signal which is filtered is sent to the $\mathrm{A} / \mathrm{D}$ converter and is converted to samples [7].

The decision-statistics can be or any value. Let us take $\mathrm{Y}^{\prime}$ as a decision-statistic [10].

$$
Y^{\prime}=\frac{1}{N_{0}} \int_{0}^{T} y_{i}^{2}(t) d t
$$

Where,

$$
\gamma=\frac{E_{s}}{N}=\frac{E_{5}}{2 N_{0}}=\frac{\lambda}{2}
$$

\subsection{AWGN Channel's detection probability}

The preset value of an energy detector is also called threshold energy defined by $\lambda[8]$. With this $\lambda$ we can evaluate 3 factors and analyze the detector performance: a) probability of missed-detection, b) the probability of false-alarm and c) the probability of detection.

The detection-probability $\mathrm{P}_{D}$ and false-alarm probability $\mathrm{P}_{F}$ are given by 


$$
\begin{aligned}
& P_{D}=P\left(Y^{\prime}>\Lambda \mid H_{1}\right) \\
& P_{F}=P\left(Y^{\prime}>\Lambda \mid H_{0}\right)
\end{aligned}
$$

Here $\Lambda$ is the threshold of detection. $\mathrm{P}_{F}$ can be written in the form of Probability density function (PDF),

$$
\begin{gathered}
P_{F}=\int_{\Lambda}^{\infty} f_{Y^{\prime}}(y) d y \\
P_{F}=\frac{1}{2^{d} \Gamma(d)} \int_{\Lambda}^{\infty} y^{d-1} \mathrm{e}^{-\left(\frac{y}{2}\right)} d y
\end{gathered}
$$

By changing the limits and substituting we get,

$$
P_{F}=\frac{1}{2 \Gamma(d)} \int_{\frac{1}{2}}^{\infty} t^{d-1} \mathrm{e}^{-(t)} d t
$$

Or

$$
P_{F}=\frac{\Gamma\left(\mathrm{d}, \frac{\Lambda}{2}\right)}{\Gamma(d)}
$$

$\Gamma($.$) , defined as an incomplete-gamma-function. Sub-$ sequently, $\mathrm{P}_{D}$ can be defined by using CumulativeDistribution-Function (CDF).

$$
P_{D}=1-F_{Y^{\prime}}(\Lambda)
$$

CDF of Y' can be obtained by [9],

$$
F_{Y^{\prime}}(y)=1-Q_{d}(\sqrt{\lambda}, \sqrt{\Lambda})
$$

Using above two equations, the $\mathrm{P}_{D}$ for $\mathrm{AWGN}$ is [11]

$$
\begin{gathered}
P_{D}=Q_{d}(\sqrt{\lambda}, \sqrt{\Lambda}) \\
P_{D}=Q_{d}(\sqrt{2} \gamma, \sqrt{\Lambda})
\end{gathered}
$$

Here $\mathrm{Q}_{d}(\ldots)$ is called the generalized Marcum-Q function. In this manner, we can calculate $\mathrm{P}_{D}$ of AWGN using the expression above.

\subsection{Probability of detection for Rayleigh Channel}

The amplitude of signal follows a Rayleigh distribution when they exists in the environment enormous number of plane waves and few types of scattering. In such cases, the SNR $\gamma$ has a PDF which is exponentially given by,

$$
f(\gamma)=\frac{1}{\bar{\gamma}} \exp ^{\left(\frac{-\gamma}{\bar{\gamma}}\right)} \quad \gamma>0
$$

We can obtain detection-probability, $\mathrm{P}_{D}$ for Rayleigh Channel by averaging their probability-density-function (PDF) over detection-probability for AWGN Channel,

$$
P_{d, R}=\int_{0}^{\infty} P_{d} f(\gamma) \mathrm{d} \gamma
$$

Here $\mathrm{P}_{D, R}$ is the probability of detection for Rayleigh Channel.

$$
P_{D, R}=\frac{1}{\bar{\gamma}} Q_{d}(\sqrt{2 \gamma}, \sqrt{\Lambda}) \mathrm{d} \gamma
$$

Simplifying[11],

$$
\begin{array}{r}
P_{D, R}=\mathrm{e}^{\left(-\frac{\Lambda}{2}\right)} \sum_{n=0}^{d-2} \frac{1}{n !}\left(\frac{\Lambda}{2}\right)^{n}+ \\
\left(\frac{1+\bar{\gamma}}{\bar{\gamma}}\right)\left[\exp ^{\left(-\frac{\Lambda}{2(1+\bar{\gamma})}\right)}-\exp ^{\left(-\frac{\Lambda}{2}\right)} \sum_{n=0}^{d-2} \frac{1}{n !}\left(\frac{\Lambda}{2(1+\bar{\gamma})}\right)^{n}\right]
\end{array}
$$

The expression above gives detection-probability $\left(\mathrm{P}_{D)}\right)$ for spectrum-sensing over Rayleigh Channel based on energy detection[8].

\section{Result}

Using MATLAB for simulation purposes for various channels (fading and non- fading) the output which was received was noted. In order to evaluate which spectrum sensing method is better various parameters are taken into accounts such as missed-detection-probability $\left(P_{m}\right)$, detection-probability $\left(P_{D}\right)$, and false-alarm-probability $\left(P_{F}\right)$. ROC curve is been plotted of $\left(P_{m}\right)$ versus $\mathrm{PF}$ and $\left(P_{F}\right)$ versus $\left(P_{F}\right)$ through which the performance can be distinguished, of various spectrum methods.

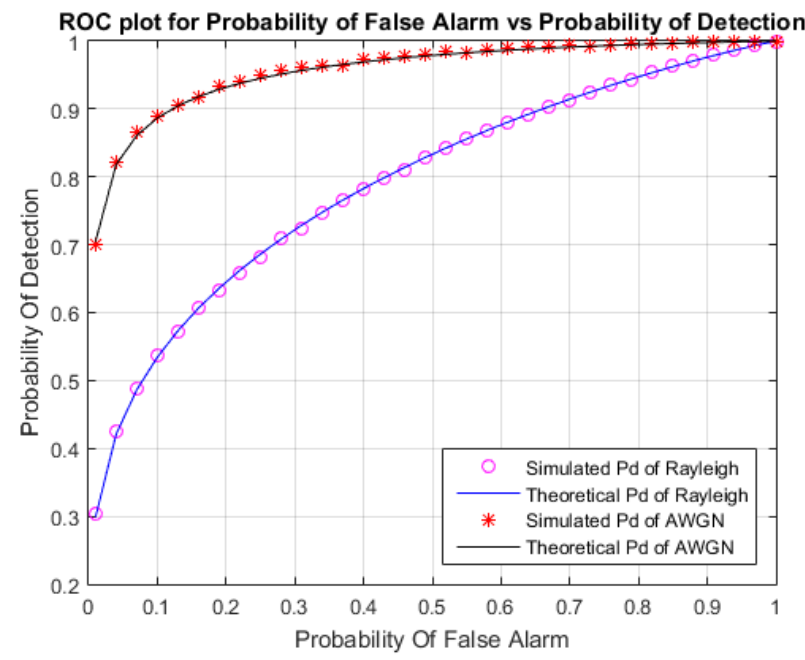

Figure 3. $P_{D}$ versus $P_{F}$ of fading and non -fading channels

From the Figure 3 it is clear that the detectionprobability (PD) of fading channel (Rayleigh) is less than the non-fading channel (AWGN). This graph clearly gives the idea that the usage of the spectrum band is less when the fading is taken into consideration. The results obtained after simulation and the theoretical ones are matched approximately. 


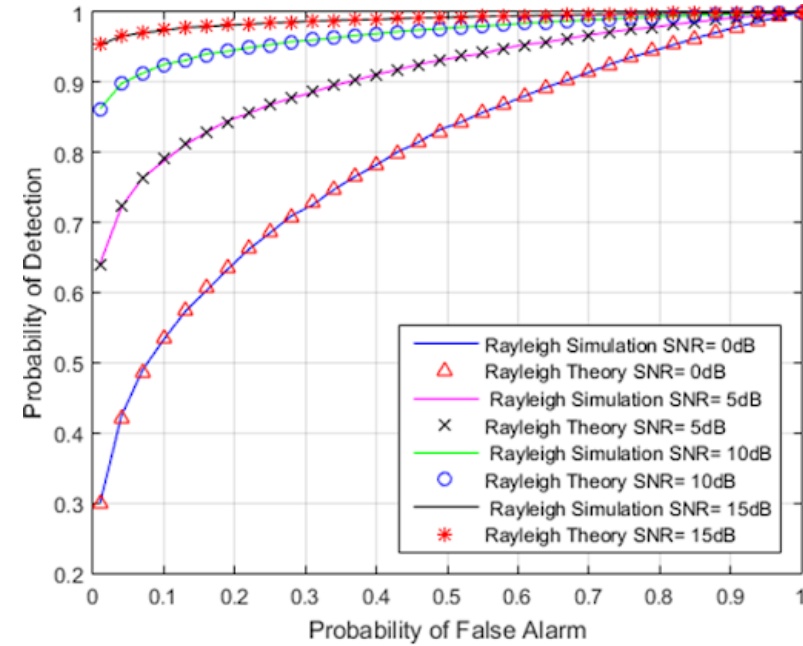

Figure 4. $P_{D}$ versus $P_{F}$ of fading channel (Rayleigh)

As from the above Figure 4 it is evident that when the energy detector is being used in such conditions that the SNR values are varied from $-30-10 \mathrm{~dB}$ the Rayleigh fading channel efficiency gets decreased accordingly. This shows that the probability detection values get increased when the signal to noise ratio increases.

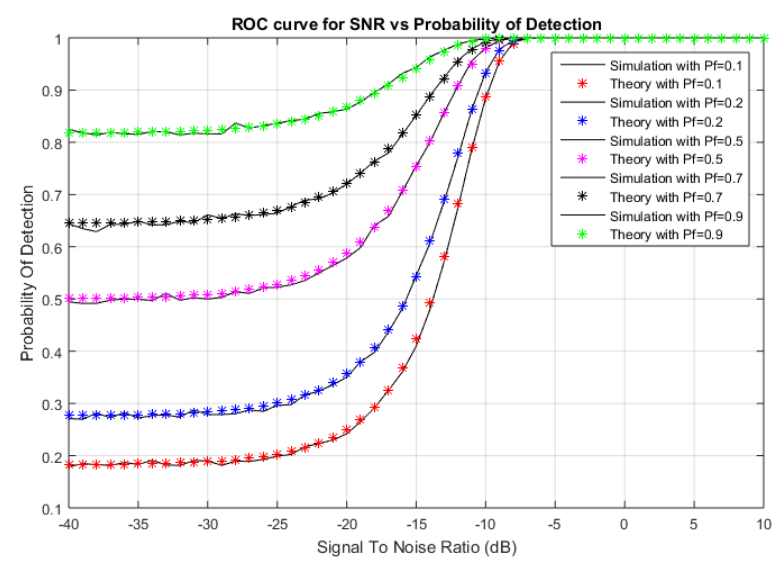

Figure 5. $P_{D}$ versus SNR of fading channel (Rayleigh)

The above Figure 5 is evident that when the probability of the false-alarm values is varied from 0.1-1 an upgrade or improvement is observed in the probability of detection.

\section{Conclusion}

In this paper, the nuts and bolts of cognitive radio have been discussed including spectrum management and performance of energy detector-based detection techniques. The closed-form expression of detection-probability and false-alarm probability over Rayleigh channel and the non-fading channel has been discussed. Different ROC curves have been plotted and the values of parameters of fading such as SNR values, false alarm probability cause a significant change in the corresponding detection probability and hence affect the overall performance. Rayleigh channel is observed in contrast with the AWGN channel. Various fading channels have varied impacts on the signal.

\section{References}

[1] J. Mitola, Cognitive radio: making software radios more personal- Volume: 6, Page numbers: 13-18, Year (1999)

[2] Simon Haykin, Cognitive radio: brain-empowered wireless communications- Volume: 23, Page numbers: 201 - 220, Year FEBRUARY(1999)

[3] Carvalho, Fabrício B S DeLopes, Waslon T Alencar, Marcelo S,"Performance of Cognitive Spectrum Sensing Based on Energy Detector in Fading Channels", Procedia Computer Science 65, 2015.

[4] Zhang S, Bao Z. Linear combination-based energy detection algorithm in low signal-to noise ratio for cognitive radios. Eur trans Telecommunication 2011;211-17.

[5] TevfikYucek and HuseyArslam,"A Survey of Spectrum Sensing Algorithms for Cognitive Radio Applications", IEEE, vol. 97,2009.

[6] Herath S, Rajatheva N, TellamburaC.Energy detection of unknown signals in fading and diversity reception. IEEE Trans communication 2007;55(1):21-4.

[7] F.F. Digham, M.S. Alouini and M.K. Simon, "Energy Detection of unknown signals over fading channels", IEEE Transactions on Communications, Vol. 5, No.1, pp. 21- 24, 2007 .

[8] M. Ranjeeth, S. Anuradha, Performance of Fading Channels on Energy Detection Based Spectrum Sensing, Procedia Materials Volume 10, 2015, Pages 361370..

[9] Patil, Vilaskumar M. et al. "Signal Detection in Cognitive Radio Networks over AWGN and Fading Channels." International Journal of Wireless Information Networks 25 (2018): 79-86.

[10] S. Atapattu, C. Tellambura and H. Jiang, "Analysis of Area under ROC curve of energy detection" IEEE Transactions On Wireless Communications, Vol. 9, 2010.

[11] Ibrahem E. Atawi, Osamah S. Badarneh, Mohammed S. Aloqlah, and Raed Mesleh. 2016. Spectrum-sensing in cognitive radio networks over composite multipath/shadowed fading channels. Comput. Electr. Eng. 52, 337-348, (May 2016). 